\title{
Article \\ Spatial Patterns of Endometriosis Incidence. A Study in Friuli Venezia Giulia (Italy) in the Period 2004-2017
}

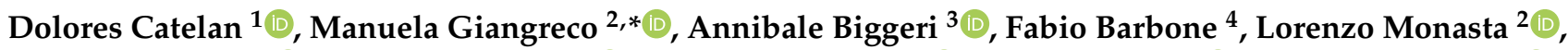

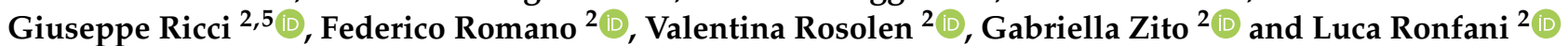

1 Unit of Biostatistics, Epidemiology and Public Health Department of Cardiac, Thoracic, Vascular Sciences and Public Health, University of Padova, 35121 Padova, Italy; dolores.catelan@unipd.it

2 Institute for Maternal and Child Health-IRCCS "Burlo Garofolo", 34137 Trieste, Italy; lorenzo.monasta@burlo.trieste.it (L.M.); giuseppe.ricci@burlo.trieste.it (G.R.); federico.romano@burlo.trieste.it (F.R.); valentina.rosolen@burlo.trieste.it (V.R.); gabriella.zito@burlo.trieste.it (G.Z.); luca.ronfani@burlo.trieste.it (L.R.)

3 Department of Statistics, Computer Science, Applications 'G. Parenti' (DiSIA), University of Florence, 50134 Firenze, Italy; annibale.biggeri@unifi.it

4 Department of Medical Area, University of Udine, 33100 Udine, Italy; fabio.barbone@uniud.it

5 Department of Medical, Surgical and Health Sciences, University of Trieste, 34149 Trieste, Italy

* Correspondence: manuela.giangreco@burlo.trieste.it; Tel.: +39-040-3785401

Citation: Catelan, D.; Giangreco, M.; Biggeri, A.; Barbone, F.; Monasta, L.; Ricci, G.; Romano, F.; Rosolen, V.; Zito, G.; Ronfani, L. Spatial Patterns of Endometriosis Incidence. A Study in Friuli Venezia Giulia (Italy) in the Period 2004-2017. Int. J. Environ. Res. Public Health 2021, 18, 7175. https:// doi.org/10.3390/ijerph18137175

Academic Editors: Milan Terzic,

Antonio Simone Laganà and

Antonio Sarria-Santamera

Received: 11 May 2021

Accepted: 29 June 2021

Published: 5 July 2021

Publisher's Note: MDPI stays neutral with regard to jurisdictional claims in published maps and institutional affiliations.

Copyright: (c) 2021 by the authors. Licensee MDPI, Basel, Switzerland. This article is an open access article distributed under the terms and conditions of the Creative Commons Attribution (CC BY) license (https:// creativecommons.org/licenses/by/ $4.0 /)$
Abstract: Background: Diagnosis of endometriosis and evaluation of incidence data are complex tasks because the disease is identified laparoscopically and confirmed histologically. Incidence estimates reported in literature are widely inconsistent, presumably reflecting geographical variability of risk and the difficulty of obtaining reliable data. Methods: We retrieved incident cases of endometriosis in women aged 15-50 years using hospital discharge records and pathology databases of the Friuli Venezia Giulia region in the calendar period 2004-2017. We studied the spatial pattern of endometriosis incidence applying Bayesian approaches to Disease Mapping, and profiled municipalities at higher risk controlling for multiple comparisons using both q-values and a fully Bayesian approach. Results: 4125 new cases of endometriosis were identified in the age range 15 to 50 years in the period 2004-2017. The incidence rate $(\times 100000)$ is $111(95 \%$ CI 110-112), with a maximum of 160 in the age group 31-35 years. The geographical distribution of endometriosis incidence showed a very strong north-south spatial gradient. We consistently identified a group of five neighboring municipalities at higher risk (RR 1.31 95\% CI 1.13; 1.52), even accounting for ascertainment bias. Conclusions: The cluster of 5 municipalities in the industrialized and polluted south-east part of the region is suggestive. However, due to the ecologic nature of the present study, information on the patients' characteristics and exposure histories are limited. Individual studies, including biomonitoring, and life-course studies are necessary to better evaluate our findings.

Keywords: endometriosis; incidence; epidemiological surveillance; disease mapping; hierarchical Bayesian models; High Risk Areas Profiling

\section{Introduction}

Endometriosis is an estrogen-dependent female chronic inflammatory disease in which endometrial tissue develops outside the uterus. [1-3] The symptoms of endometriosis are chronic pelvic pain, fatigue, dysmenorrhea, dyspareunia, abnormal/irregular uterine bleeding and infertility or subfertility, as response of endometrial tissue to hormonal stimulation $[4,5]$.

Endometriosis causes high social and healthcare system costs, worsening quality of life and work productivity. The average time from onset of symptoms to diagnosis ranges from 6 to 12 years, delaying appropriate therapy $[6,7]$.

Diagnosing endometriosis also depends on the gynecologist and the assessment method used. Identifying cases in the general population from endometriosis registers is 
difficult because laparoscopic visualizations and histologic confirmation can only obtain a definitive diagnosis of the disease according to the ESHRE (European Society of Human Reproduction and Embryology) guidelines [8]. Laparoscopy is an invasive surgical procedure that is indicated in women with clear symptoms $[9,10]$. However, many women are asymptomatic, or there may be an overlap of symptoms with other conditions and lesions that might heal spontaneously or following hormonal treatment without a previously made diagnosis [11,12].

The estimated incidence of endometriosis reported in the literature shows considerable variability. It is difficult to interpret, reflecting geographical variability of risk or simply due to the difficulty mentioned above of obtaining reliable data [4,11,13-17].

In a previous paper [18], we estimated the incidence and prevalence of endometriosis in women between 15-50 years, in the Friuli Venezia Giulia (FVG) region, in the period 2011-2013. We found an endometriosis incidence rate of $0.11 \%$ and a prevalence of $1.82 \%$.

When risk factors for disease are unknown, maps may help highlight spatial trends in the distribution of relative risk and generate etiological hypotheses.

However, when the inferential goal is to identify municipalities with risk diverging from the local or national reference, the problem must be addressed with a multiple test approach, testing hypothesis departure from the reference null for each municipality.

In public health implications of disease mapping, several different decision rules are discussed [19]. However, in practice, despite some criticism, $p$-values are still used to scrutinize long lists of relative risks.

In functional Genomics data analysis and other high-throughput biological areas of application, control of the False Discovery Rate (FDR) has become popular for multiple comparisons adjustment $[20,21]$. To date, limited research has been conducted on FDR in disease mapping [22].

In the Bayesian context, posterior probabilities of RR greater than the null obtained from hierarchical Bayesian models failed to address the multiple comparison problem [2325]. As an alternative, classification probabilities obtained from Bayesian mixture models have been adjusted for multiple comparisons [26] and proposed in the context of disease mapping [27].

The goals of our study were to update the results reported in our previous paper [18]. We also sought to describe the spatial pattern of the incidence of endometriosis in FVG in the period 2004-2017 using the two most common Bayesian approaches of Disease Mapping. We also aimed to identify municipalities at different risk of endometriosis, controlling for multiple comparisons using both "frequentist" FDR and a fully Bayesian approach.

\section{Materials}

\subsection{Incidence of Endometriosis}

Data were extracted from the FVG Regional Repository of MicroData (RRMD) by record linkage using a unique anonymous identifier. RRMD is a centralized record system automatically pooling health care data from the national health service. The FVG Regional Health Authority granted access to the data, so no approval was required from the institutional review board of our Institute. FVG is a region located in the northeast of Italy. It covers $7855 \mathrm{Km}^{2}$ and has a population of approximately 1.22 million people, of which 630 thousand are women.

All endometriosis cases were extracted from hospital discharge and anatomic pathology databases for the years 2004-2017. We used the International Classification of Diseases, Ninth Revision ICD-9, codes 617.0-617.9, to identify at least one hospitalization with the diagnosis of endometriosis in the hospital discharge database. In contrast, we used the Systematized Nomenclature of Medicine, SNOMED (codes M-89311, D7-72000, D7-72010, D772020, D7-72100, D7-72106, D7-72110, D7-72114, D7-72120, D7-72122, D7-72124, D7-72126, D7-72130, D7-72132, D7-72134, D7-72140, D7-72142, D7-72144, D7-72146, D7-72160, D772170, D7-72180, D7-72190), to identify endometriosis in the anatomic pathology database.

We selected women 15-50 years of age residing in the FVG region. 
We considered as case a woman with:

1. At least one hospitalization with a diagnosis of endometriosis associated with the identification of endometriosis from the anatomic pathology database. The association was via a unique anonymous identifier of women and temporal proximity.

2. At least one hospitalization with a diagnosis of endometriosis confirmed by laparoscopy or a surgical procedure allowing direct visualization.

3. Identification of endometriosis from anatomic pathology database, without hospitalization with a diagnosis of endometriosis.

Patients with diagnoses supported by imaging procedures alone (i.e., ultrasound, magnetic resonance, computerized axial tomography) were excluded.

To identify incident cases, we included only patients without a diagnosis of endometriosis, based on the criteria described above, in the previous 10 years.

We calculated for each calendar year incidence rates with the number of endometriosis cases in the age range 15 to 50 as the numerator and the number of women aged 15 to 50 residing in FVG, derived from the Italian National Institute of Statistics, [28] as the population-time denominator. We then stratified the incidence rates by age classes, classifying cases based on their age at diagnosis in 15-20, 21-25, 26-30, 31-35, 36-40, 41-45, 46-50.

The analyses were performed using SAS software, Version 9.4 (SAS Institute Inc., Cary, NC, USA).

\subsection{Standardized Incidence Ratios}

Following internal indirect standardization and classification of the population in 7 age classes (15-20, 21-25, 26-30, 31-35, 36-40, 41-45, 46-50), a set of reference rates (Friuli Venezia Giulia region, 2004-2017) were used to compute the expected number of cases for each municipality.

For each i-th municipality $(i=1, \ldots, 216)$, we calculated the standardized incidence ratio (SIR = observed/expected number of cases) as an estimate of the relative risk (RR), i.e., the disease risk in each area compared to the adopted standard. In this way, we implicitly specified the same null hypothesis $\mathrm{H}_{0}: \mathrm{RR}_{\mathrm{i}}=1$, testing the procedure for each area through indirect standardization [29].

\section{Methods}

\subsection{Models for Disease Mapping}

We used the two most common Bayesian hierarchical models for Disease Mapping: the Poisson-Gamma model [30] and the Besag-York-Mollié (hereafter known as BYM) model [31]. The objective of these models is to account for overdispersion and stabilize relative risk estimates.

In detail, let's assume that the observed number of incident cases of endometriosis in the i-th municipality $Y_{i}(i=1, \ldots, 216)$ follows a Poisson distribution with mean $E_{i} x$ $\theta_{i}$, where $E_{i}$ is the expected number of cases under indirect standardization and $\theta_{i}$, is the relative risk. The maximum likelihood estimator of $\theta_{\mathrm{i}}$ is called the standardized incidence ratio (SIR: $\hat{\vartheta}_{i}=Y_{i} / E_{i}$ ).

Bayesian inference requires the specification of appropriate prior distributions on model parameters.

Clayton and Kaldor [30] assumed a Gamma $(k, v)$ prior distribution for $\theta_{\mathrm{i}}$. The hyperparameters $\mathrm{k}$ and $v$ are assumed to be exponentially distributed. Poisson random variability is filtered out in this model, and relative risk estimates are shrunken toward the general mean.

Besag et al. [31] specified a random effect $\log$-linear model for the relative risk $\log \left(\theta_{\mathrm{i}}\right)$ $=u_{i}+v_{i}$. The heterogeneity random term $u_{i}$ represents an unstructured spatial variability component assumed a priori distributed as $\operatorname{Normal}\left(0, \lambda_{\mathrm{u}}\right)$.

The clustering random term $\mathrm{v}_{\mathrm{i}}$ represents the structured spatial variability component assumed to follow a priori an intrinsic conditional autoregressive (ICAR) model. In other words, denoting $S_{i}$ as the set of the areas adjacent to the $i$-th one, $v_{i} \mid v_{j} \in S i$ is assumed 
distributed as $\operatorname{Normal}\left(\bar{v}_{i}, \lambda_{\mathrm{v}} \mathrm{n}_{\mathrm{i}}\right)$ where $\bar{v}_{i}$ is the mean of the terms of areas adjacent to the $i$-th one [32] and $\lambda_{V} n_{i}$ is the precision which is dependent on $n_{i}$, the number of areas in $S_{i}$. The hyperprior distributions of the precision parameters $\lambda_{v}, \lambda_{u}$ are assumed to be Gamma $(0.5,0.0005)[33]$.

The BYM model shrinks the relative risk estimates both toward the local and the general mean through these two random terms.

\subsection{Profiling Municipalities at High Risk}

Identifying the municipalities at high risk leads us in a multiple comparison framework since we have $\mathrm{m}$ test of hypothesis $(\mathrm{m}=216$, number of municipalities in the region). We control for uncertainty due to multiplicity.

\subsubsection{Simple Approaches Based on $p$-Values}

The commonly controlled quantity to account for multiple testing is the Family Wise Error Rate (FWER), and the most common method is the Bonferroni approach. Benjamini e Hochberg [34] proposed a procedure to control the proportion of false rejections among the total number of rejections. They called this quantity the False Discovery Rate (FDR), which is more appropriate in our context since we are performing $\mathrm{m}$ identical tests with $\mathrm{m}$ different implications if the null hypothesis would be rejected [22]. Storey [20] proposed an exploratory use of the positive FDR (pFDR), i.e., the FDR conditional to having at least one rejection. The $\mathrm{pFDR}$ can be interpreted as a posterior Bayesian probability and can be used to define the q-value, Prob $\left(\mathrm{H}_{0} \mid \mathrm{T} \geq \mathrm{T}_{\mathrm{obs}}\right)$, $\mathrm{T}$ being a test statistic, and $\mathrm{T}_{\mathrm{obs}}$ the observed value, for a generic $\mathrm{i}$-th test. The q-value is the minimum pFDR in which we can incur in the rejection of the null hypothesis based on the observed or more extreme values of the test statistics. The q-value is a measure that takes multiple testing into account. The "frequentist" calculation of q-values is based on ordered $p$-values and is reported in Reference [20]. In [20], an empirical Bayesian procedure is proposed. When profiling is the study's goal, as in our case, it can be useful to screen each area using the q-values instead of simply classifying them as "significant/non-significant" according to the assumed level of FDR that is being controlled [35].

For a single hypothesis test, Goodman [36] proposed using the minimum Bayes factor to evaluate how far the observed data moves us from an initial null state. Briefly, if we consider two hypotheses $\mathrm{H}_{0}$ and $\mathrm{H}_{1}$, and the data $\mathrm{Y}$, the Bayes theorem implies

$$
\frac{P(H 0 \mid Y)}{P(H 1 \mid Y)}=\frac{P(Y \mid H 0)}{P(Y \mid H 1)} \frac{P(H 0)}{P(H 1)}
$$

where $\frac{P(H 0)}{P(H 1)}$ is the prior Odds, $\frac{P(Y \mid H 0)}{P(Y \mid H 1)}$ is the Bayes Factor (BF) and $\frac{P(H 0 \mid Y)}{P(H 1 \mid Y)}$ is the posterior Odds. The BF quantifies the evidence of data $\mathrm{Y}$ for $\mathrm{H}_{0}$ vs. $\mathrm{H}_{1}$.

The minimum Bayes factor is the smallest possible Bayes factor for the point null hypothesis against the alternative within the specified class of alternatives.

Edwards et al. [37] show that if $\mathrm{H}_{0}: \mu=\mu_{0}, \mathrm{Y} \sim \operatorname{Normal}\left(\mu, \sigma^{2}\right)$ then $\mathrm{BF}=\frac{P(Y \mid H 0)}{P(Y \mid H 1)} \geq$ $\exp \left(-0.5 t^{2}\right)$ where $t=\left(y-\mu_{0}\right) / \sigma$ is the number of standard errors from the null value. Sellke et al. [38] proposed an approach that works directly with $p$ values.

For our analysis, we used two previously described approaches based on the transformation of $p$-values into Bayesian posterior probabilities of the null. The q-values, when considering the whole set of observations accounting for multiple comparisons, and the posterior probabilities obtained from the minimum Bayes factor under three null prior probabilities (optimistic with odds 1:3, neutral 1:1, pessimistic 3:1).

The analyses were performed using STATA software version 14 (StataCorp, College Station, TX, USA). 


\subsubsection{Hierarchical Bayesian Mixture Model}

When selective inference, aiming to identify divergent areas, is the goal, the Bayesian models used for Disease mapping (Section 3.1) are further complicated by introducing a third level into the hierarchy, assuming a mixture model for the unknown relative risks $\theta_{\mathrm{i}}[27]$.

The likelihood for $Y_{i}$ is still Poisson $\left(E_{i} \theta_{i}\right)$, where $E_{i}$ is the expected number of cases and $\theta_{\mathrm{i}}$ the relative risk in the $\mathrm{i}$-th municipality. We then assume that $\log \left(\theta_{\mathrm{i}}\right)=\mathrm{r}_{\mathrm{i}} \mu_{0 \mathrm{i}}+$ $\left(1-r_{i}\right) \mu_{1 i}$ where, i.e., the logarithm of the relative risk $\theta_{i}$ is modeled as the mixture of two components: $\mu_{0 \mathrm{i}}$, the value of the log relative risk under the null hypothesis $\mathrm{H}_{0}$, and $\mu_{1 i}$ the corresponding value under the alternative $H_{1}$. The $r_{i}$ indicator denotes the group membership.

Under the null $\mathrm{H}_{0}$ we assume that all the probability mass is concentrated at one point, i.e., $\mu_{0 \mathrm{i}}=0$, leaving only a Poisson random variability. Under the alternative $\mathrm{H}_{1}$ extra Poisson variability, reflecting the heterogeneity of relative risk among areas is modeled according to the Poisson Gamma or the BYM models.

The prior distribution for the indicator of the unknown true status, $r_{i}$, is assumed to be Bernoulli distributed with parameter $\pi_{\mathbf{i}}$, which, in turn, is modeled as Beta $\left(\alpha_{1}\right.$, $\alpha_{2}$ ) distribution.

The quantity of interest for each i-th area is some appropriate summary measure over the posterior distribution of $\pi_{\mathrm{i}}$-i.e., the posterior classification probability to belong to the null hypothesis set. The term "classification probabilities" underlines the connection to classification theory and clearly distinguishes it from Prob $\left(\theta_{i}>1 \mid Y\right)$, denoted as posterior probabilities $[39,40]$.

The a priori distribution for $\pi_{\mathrm{i}}$ is assumed to be an exchangeable informative Beta $\left(\alpha_{1}\right.$, $\alpha_{2}$ ). Changing the value of the Beta parameters $\alpha_{1}, \alpha_{2}$, we a priori introduced in the model our prior belief on the percentage of divergent areas, consistent with the non-Bayesian simple approaches presented in Section 3.2.1.

All the Bayesian analyses were performed using the WinBugs1.4 software [41].

We ran two independent chains for each model, and the convergence of the algorithm was performed following Reference [42]. We discarded the first 100,000 iterations (burn-in) and stored for estimation 50,000 iterations.

\subsection{Sensitivity Analysis}

To compare areas at higher and lower risk within the region for explanatory variables, we planned to use the Chi-square test for categorical variables and the non-parametric WilcoxonMann-Whitney test for continuous variables (data shown in Supplementary File S1: Table S1).

We used capture-recapture methods to evaluate the coverage of the different registries (hospital discharges and anatomic pathology) and the presence of possible ascertainment bias [43].

\section{Results}

For the period 2004-2017, 4125 new cases of endometriosis were identified in the age range 15 to 50 (Table 1$)$. The crude incidence rate $(\times 100,000)$ of endometriosis in women aged 15-50 years for the period 2004-2017 was 111, if we consider all diagnoses, with and without histological confirmation (Table 1). The age-specific incidence rate of endometriosis was highest in the age group 31-35 $(160 \times 100,000)$.

Of the 4125 cases, 1471 (35.7\%) were hospitalizations with a diagnosis of endometriosis associated with the identification of endometriosis from the anatomic pathology database. Of this, $1846(44.8 \%)$ were hospitalizations with a diagnosis of endometriosis confirmed by laparoscopy or other surgical procedure allowing direct visualization, and $808(19.6 \%)$ were identified only from the anatomic pathology database. 
Table 1. Age-specific incidence of endometriosis in women residing in FVG in the years 2004-2017.

\begin{tabular}{ccc}
\hline Age & $\begin{array}{c}\text { Women Residing in the } \\
\text { Region * }\end{array}$ & $\begin{array}{c}\text { Endometriosis } \\
\left.\text { n (Rates } \times \mathbf{1 0}^{\mathbf{5}}\right)\end{array}$ \\
\hline $15-20$ & 402,526 & $48(12)$ \\
$21-25$ & 365,815 & $228(62)$ \\
$26-30$ & 442,259 & $631(143)$ \\
$31-35$ & 541,962 & $869(160)$ \\
$46-40$ & 633,878 & $846(133)$ \\
$46-50$ & 681,123 & $833(122)$ \\
total 15-50 & 654,892 & $670(102)$ \\
\hline
\end{tabular}

* Numbers represent the sum of women residing in the region in the fourteen years considered.

\subsection{Disease Mapping}

The SIRs in the period 2004-2017 range between 0 and 3.5 (Figure 1A). The geographical distribution is heterogeneous (Figure 1B) with high/low risk areas. The spatial pattern was more evident if we considered the smoothed map of SIR under the two Bayesian models (Poisson-Gamma and BYM) (Figure 2A,B). The shrinking effect of the Bayesian estimators was evident when comparing it to the SIR map (Figure 1B). Relative risks (RR) of areas with few expected counts and extreme SIR were regressed to the mean. Since we used indirect standardization with FVG reference rates, the observed mean was close to the null RR of one. In the BYM model, the relative importance of the clustering component compared to the heterogeneity component, i.e., the ratio of variances of the two random terms is $4: 1$. Overall, the geographical pattern of the incidence of endometriosis showed a very strong spatial structure, with southern areas at higher risk.

\subsection{Profiling Municipalities at High Risk}

In Figure 3A, we report the funnel plot [44], a graph of the effect measure (SIR in our case) against its precision (the precision of the SIR is proportional to $E_{i}$ since the asymptotic variance $\left.(\mathrm{SIR})=1 / \mathrm{E}_{\mathrm{i}}\right)$. This plot shows that the precision in estimating the risk will increase as the population dimension of the area increases. SIR from small areas will spread widely in the diagram, while the variability will be narrower for more populated areas. If the null hypothesis is true for all areas, the plot would be a symmetric funnel centered on the reference line at an ordinate null value of one.

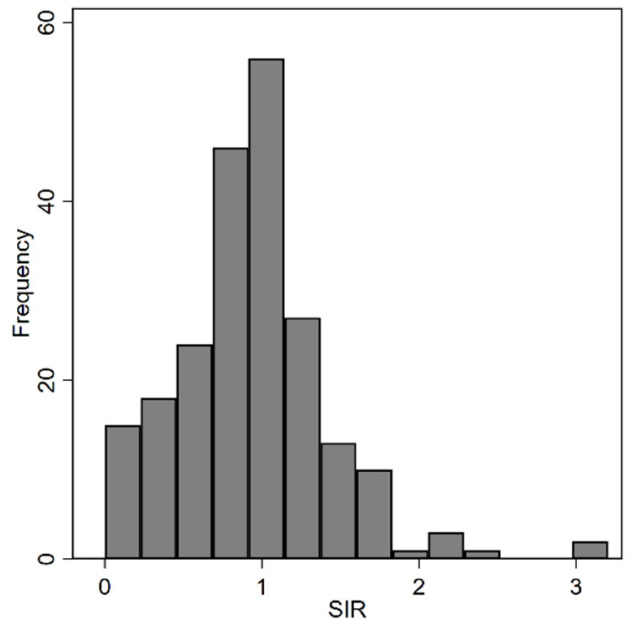

(A)

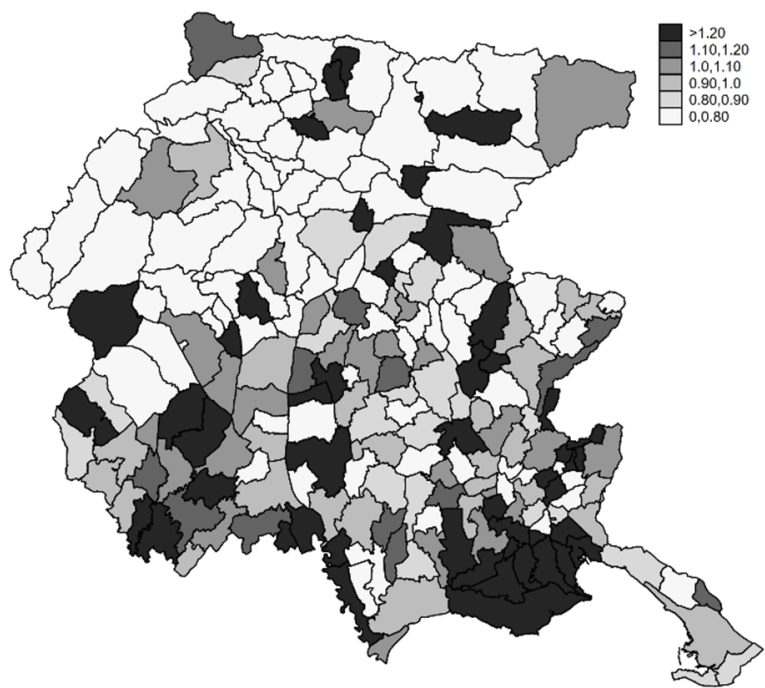

(B)

Figure 1. (A) Histogram of SIRs (B) Spatial distribution of SIRs. FVG, 2004-2017. 


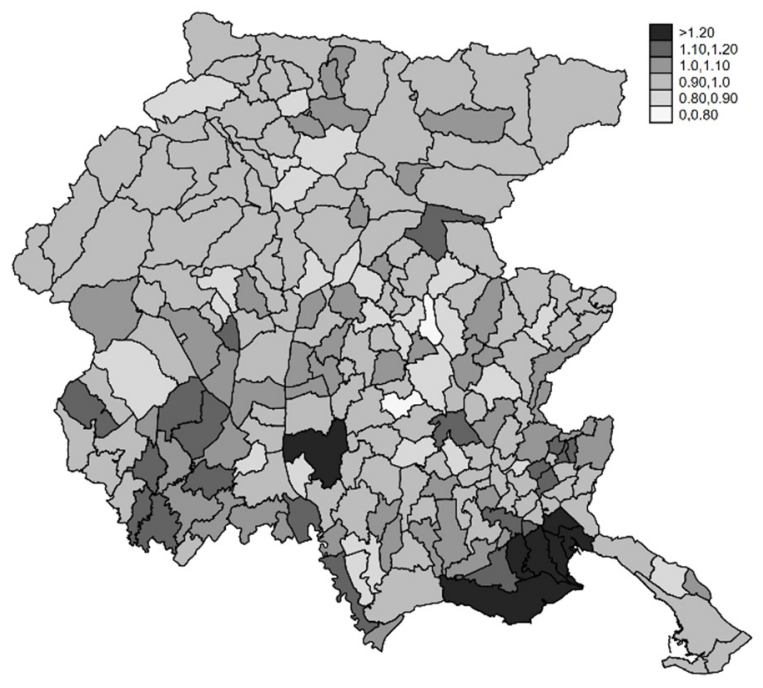

(A)

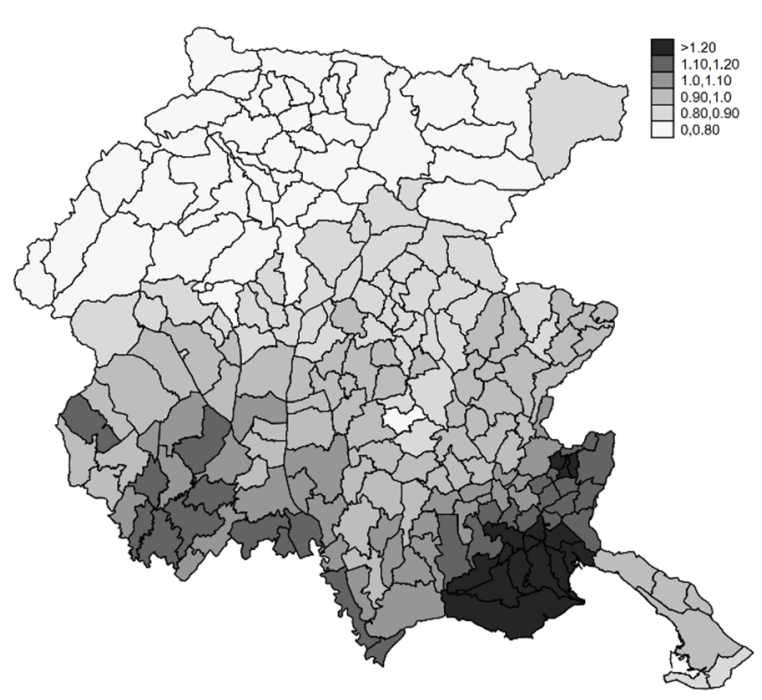

(B)

Figure 2. Posterior relative risk estimates from the Poisson-Gamma (A) and BYM (B) models (see text). FVG, $2004-2017$.

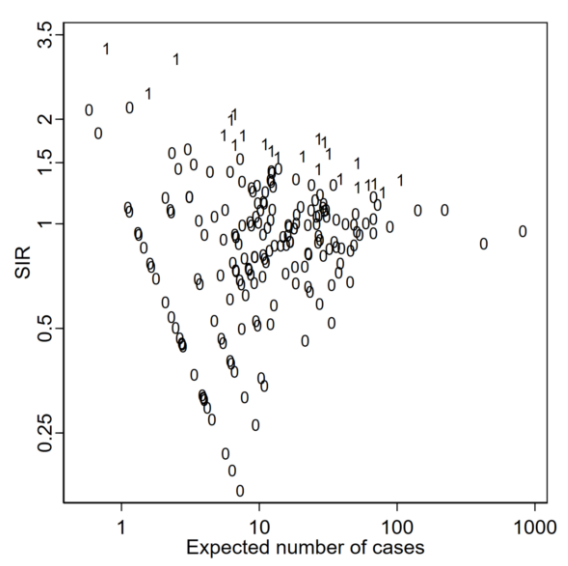

(A)

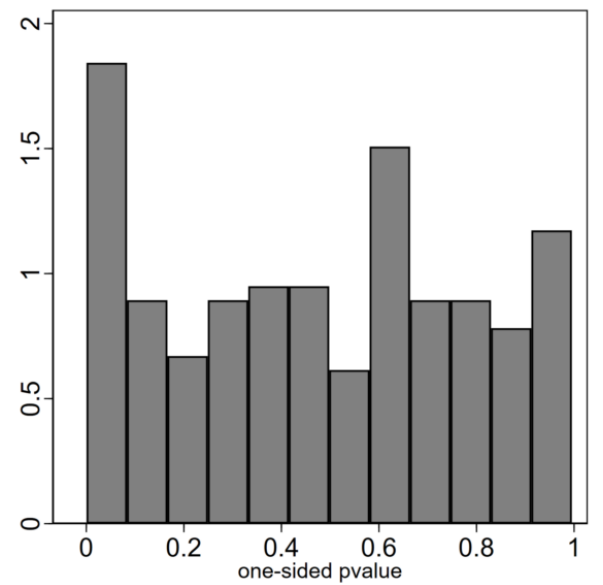

(B)

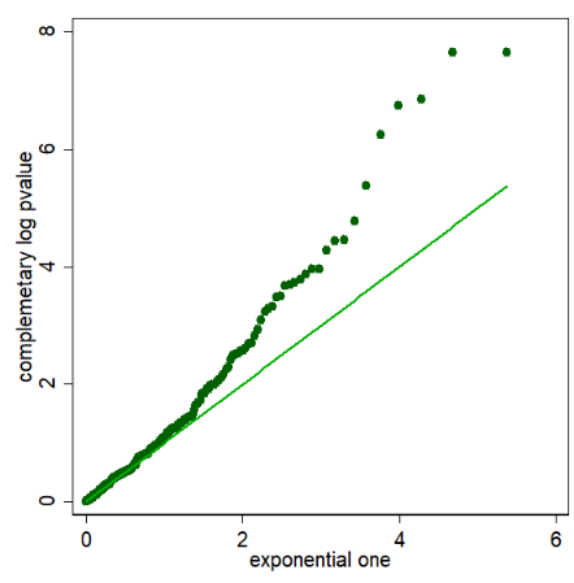

(C)

Figure 3. Funnel plot of SIRs: label 1 indicates areas with one-sided $p$-value $<0.05$ (A); Histogram of empirical one-sided $p$-values * (B); Quantile-Quantile plot of complementary log empirical $p$-values versus theoretical exponential (1) (C). FVG, 2004-2017. * For each area out of $\mathrm{m}$, the one-sided $p$-value Prob ( $\mathrm{Y} \geq$ Yobs $\mid \mathrm{H} 0)$ under the null hypothesis H0: $\theta=1$ against the alternative H1: RR $>1$ is obtained from the exact Poisson distribution.

Figure $3 \mathrm{~A}$ plotted the municipalities with one-sided $p$-values $<0.05$, identified with label 1. Figure 3B shows the empirical distribution of the one-sided $p$-values. If no areas diverged, the $p$-value distribution would be uniform. There is evidence of departure from the null because the relative frequency of small $p$-values is greater than expected under the uniform distribution.

Figure $3 \mathrm{C}$ shows the quantile-quantile plot of the complementary log transformation of empirical $p$-values against the null theoretical exponential (1) distribution. The plot was useful for identifying the outlying observations responsible for the departure from the uniform distribution shown in panel 3B.

To screen these divergent areas according to a multiple testing correction, we report in Table 2 the municipalities with one-sided $p$-values $<0.05$ and the corresponding q-values. 
Table 2. Municipalities with one-sided $p$-value $<0.05$, number of cases, SIR, $p$-value, FDR (q-value), calibrated Goodman $p$-values under 3 alternative prior odds between the null and alternative hypothesis $(3: 1 ; 1: 1 ; 1: 3)$. FVG $2004-2017$.

\begin{tabular}{|c|c|c|c|c|c|c|c|}
\hline \multirow[b]{2}{*}{ Municipality } & \multirow[b]{2}{*}{ Number of Cases } & \multirow[b]{2}{*}{$\operatorname{SIR}^{\mathrm{a}}$} & \multirow[b]{2}{*}{$p$-Value } & \multirow[b]{2}{*}{ q-Value } & \multicolumn{3}{|c|}{ Decrease in Probability of the Null Hypothesis } \\
\hline & & & & & $\begin{array}{l}\text { From } 75 \% \text { to } \\
\text { No Less than }\end{array}$ & $\begin{array}{l}\text { From } 50 \% \text { to } \\
\text { No Less than }\end{array}$ & $\begin{array}{l}\text { From } 25 \% \text { to } \\
\text { No Less than }\end{array}$ \\
\hline San Canzian d'Isonzo & 38 & 1.759 & 0.0005 & 0.0522 & 0.0127 & 0.0043 & 0.0014 \\
\hline Staranzano & 41 & 1.720 & 0.0005 & 0.0522 & 0.0128 & 0.0043 & 0.0014 \\
\hline Ronchi dei Legionari & 62 & 1.498 & 0.0011 & 0.0640 & 0.0260 & 0.0088 & 0.0030 \\
\hline Monfalcone & 114 & 1.339 & 0.0012 & 0.0640 & 0.0287 & 0.0098 & 0.0033 \\
\hline Grado & 41 & 1.595 & 0.0019 & 0.0836 & 0.0442 & 0.0152 & 0.0051 \\
\hline Lusevera & 6 & 2.986 & 0.0046 & 0.1672 & 0.0924 & 0.0328 & 0.0112 \\
\hline San Lorenzo Isontino & 11 & 2.073 & 0.0085 & 0.2619 & 0.1480 & 0.0547 & 0.0189 \\
\hline Fiumicello & 26 & 1.565 & 0.0117 & 0.2858 & 0.1864 & 0.0709 & 0.0248 \\
\hline Codroipo & 72 & 1.307 & 0.0119 & 0.2858 & 0.1892 & 0.0722 & 0.0253 \\
\hline Mariano del Friuli & 10 & 1.998 & 0.0138 & 0.2982 & 0.2096 & 0.0812 & 0.0286 \\
\hline Morsano al Tagliamento & 15 & 1.697 & 0.0190 & 0.3253 & 0.2587 & 0.1042 & 0.0373 \\
\hline Latisana & 64 & 1.295 & 0.0191 & 0.3253 & 0.2593 & 0.1045 & 0.0374 \\
\hline Gradisca d'Isonzo & 31 & 1.438 & 0.0209 & 0.3253 & 0.2743 & 0.1119 & 0.0403 \\
\hline Capriva del Friuli & 11 & 1.798 & 0.0229 & 0.3253 & 0.2900 & 0.1198 & 0.0434 \\
\hline Turriaco & 16 & 1.624 & 0.0239 & 0.3253 & 0.2977 & 0.1238 & 0.0450 \\
\hline Prata di Pordenone & 42 & 1.350 & 0.0249 & 0.3253 & 0.3047 & 0.1275 & 0.0464 \\
\hline Barcis & 2 & 3.203 & 0.0256 & 0.3253 & 0.3096 & 0.1300 & 0.0475 \\
\hline Polcenigo & 17 & 1.557 & 0.0303 & 0.3528 & 0.3401 & 0.1466 & 0.0542 \\
\hline Cordenons & 78 & 1.233 & 0.0310 & 0.3528 & 0.3448 & 0.1492 & 0.0552 \\
\hline Fiume Veneto & 53 & 1.276 & 0.0359 & 0.3865 & 0.3726 & 0.1652 & 0.0619 \\
\hline Arba & 8 & 1.801 & 0.0376 & 0.3865 & 0.3811 & 0.1703 & 0.0640 \\
\hline Dolegna del Collio & 3 & 2.378 & 0.0394 & 0.3865 & 0.3901 & 0.1758 & 0.0664 \\
\hline Mossa & 9 & 1.689 & 0.0454 & 0.4263 & 0.4179 & 0.1931 & 0.0739 \\
\hline
\end{tabular}

${ }^{\text {a }}$ SIR = Standardized Incidence Ratio.

Of the 216 areas examined, 23 have $p$-values $<0.05$. Applying Bonferroni's correction (with a probability of type I error set at $5 \%$ ), no areas would be divergent from the null. Using Storey's q-value, 6, 4, and 2 areas were selected when the threshold was set at 20\%, $10 \%$, and $5 \%$, respectively.

The last three columns of Table 2 report the calibrated Goodman $p$-values under three different prior odds $\frac{P(H 0)}{P(H 1)} 3: 1 ; 1: 1 ; 1: 3$. This analysis is appropriate if we assume the point of view of each area separately, considering any multiple comparisons adjustment not pertinent. Even with a high probability of the null compared to the alternative, the posterior probability of the null was less than $5 \%$ for five municipalities.

In Figure 4, we report the posterior probabilities Prob $\left(\theta_{i}>1 \mid Y\right)$ obtained from the Poisson Gamma (A) and the BYM (B) models. The maps are coherent with the distribution of the RR, with areas at high posterior probabilities located in the southwest part of the region. However, considering that such Posterior probabilities are not adjusted for multiplicity, they are useful to describe the overall spatial risk pattern. 


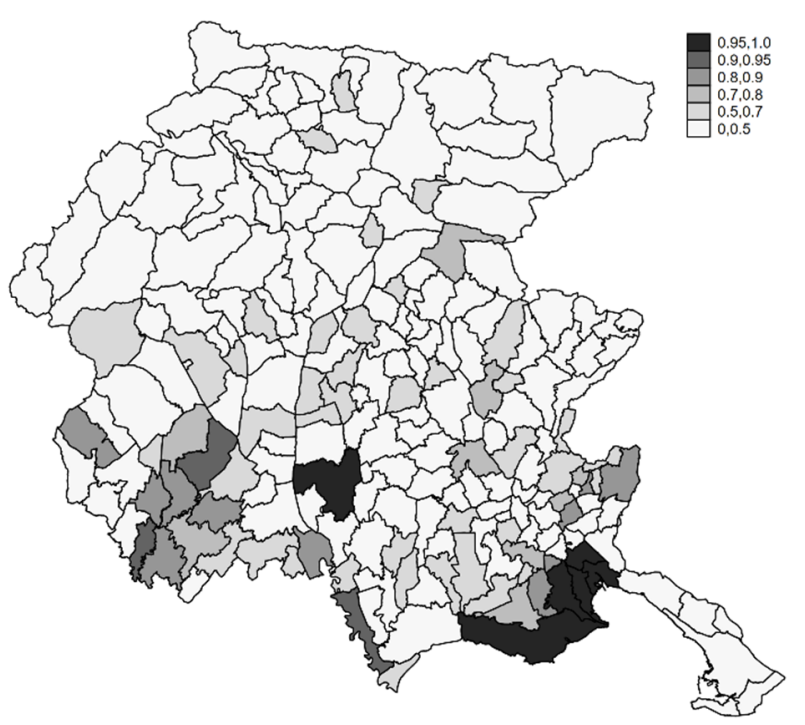

(A)

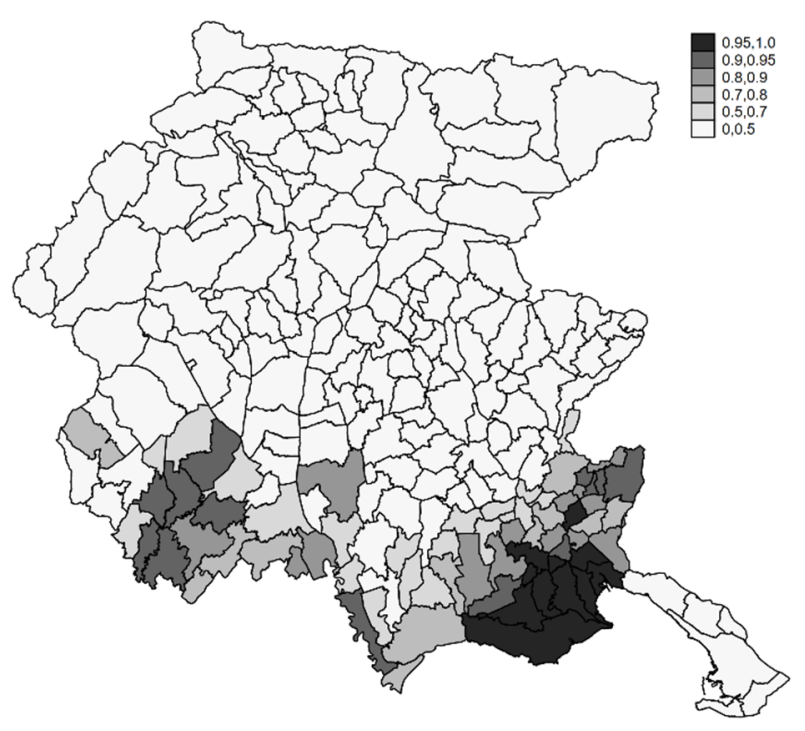

(B)

Figure 4. The posterior probability of RR > 1 from the Poisson-Gamma (A) and BYM (B) models. FVG, 2004-2017.

The complex Bayesian tri-level modeling embeds the previous points of view in a unified perspective. In Figure 5, we map posterior inclusion probabilities (i.e., the complement to the posterior classification probabilities Prob $\left(r_{i}=0 \mid Y>Y_{i} ; Y\right)=1-\operatorname{Prob}$ $\left(r_{i}=1 \mid Y=Y_{i} ; Y\right)$ [42] under the mixture Bayesian Poisson-Gamma (A, C) and BYM models (B, D). In the first row, inclusion probabilities were obtained specifying a Beta $(2.5,7.5)$ as a priori for $\pi_{i}$, that is a Beta with an expected value of $25 \%$, that are sensible for a prior belief of a percentage of divergent areas around $75 \%$. In the second row, we specified a Beta $(7.5,2.5)$ for $\pi_{i}$, which is a Beta with an expected value of $75 \%$, meaning a prior belief of a percentage of divergent areas around $25 \%$. The results were sensitive to prior choices for $\pi_{\mathrm{i}}$ with the Poisson Gamma model. Under the BYM specification, the results were consistent with those obtained by the simpler approaches, and five areas were consistently identified as divergent.

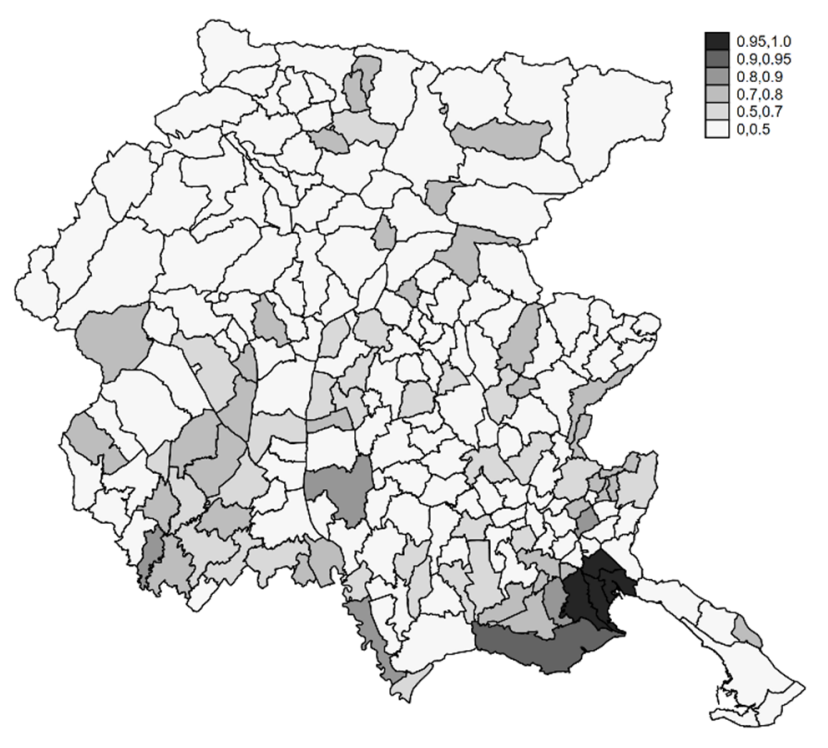

(A)

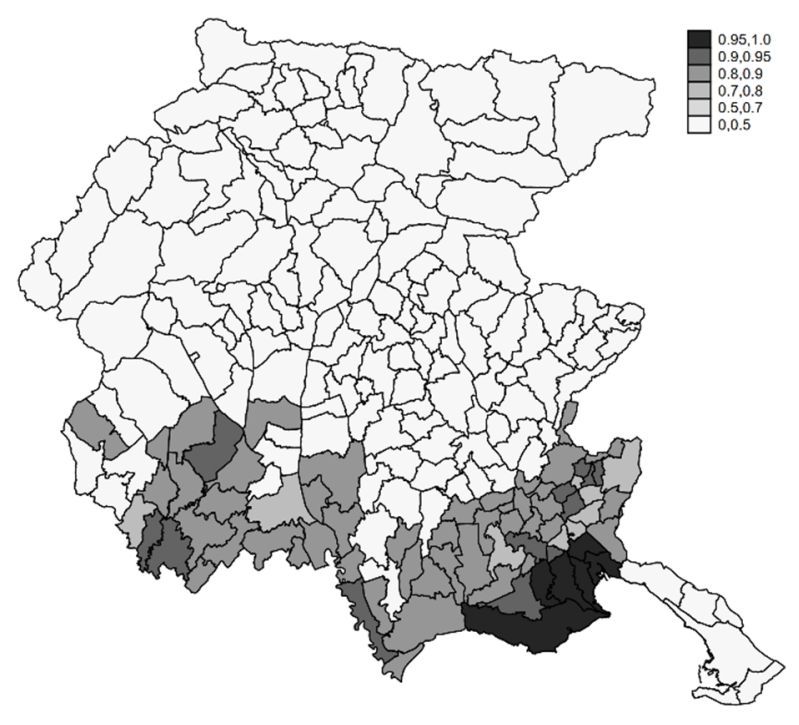

(B)

Figure 5. Cont. 


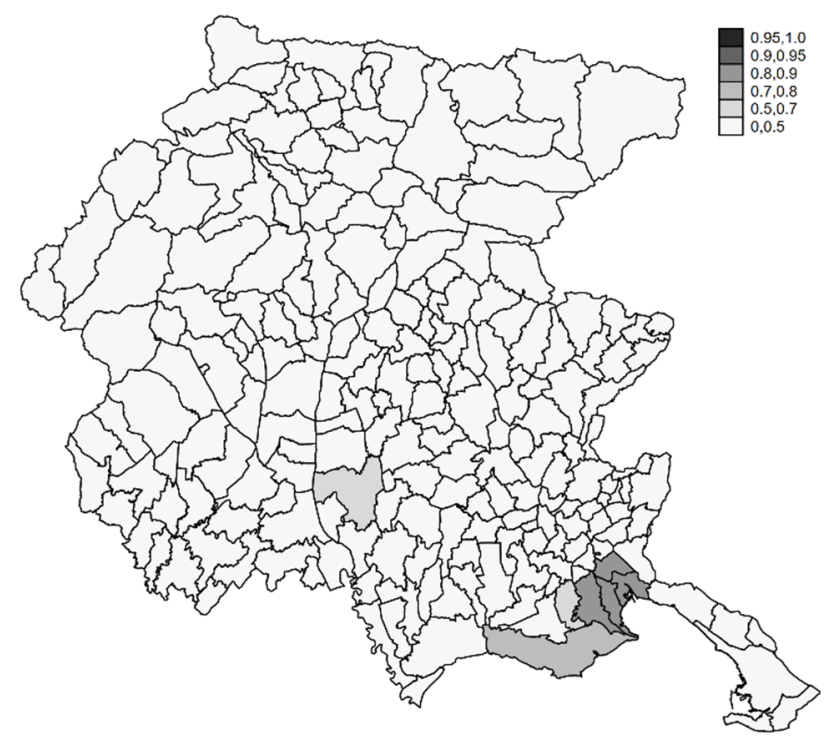

(C)

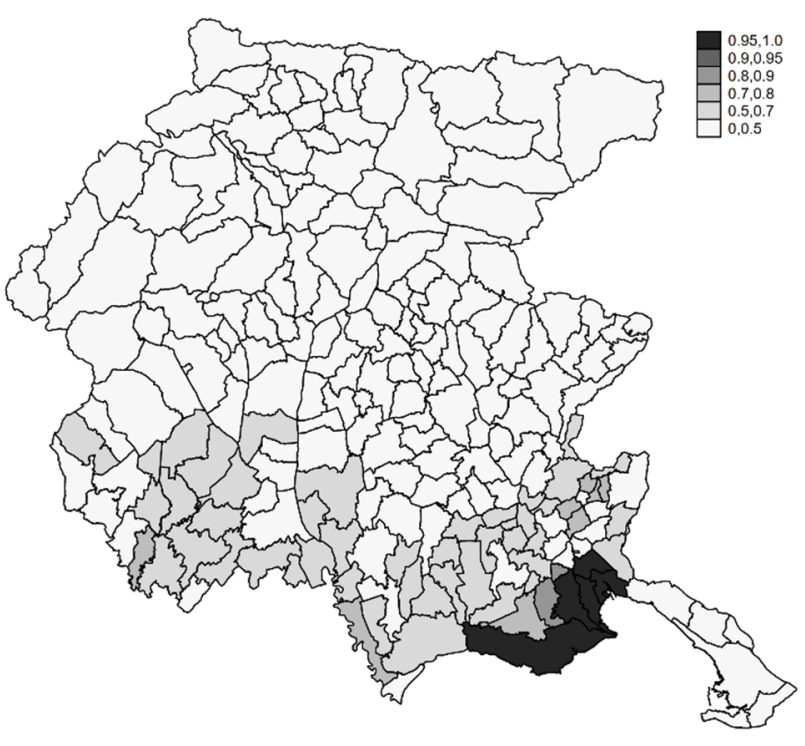

(D)

Figure 5. Posterior inclusion probabilities under the tri-level Poisson-Gamma under a priori P(H0): 25\% (A); Posterior inclusion probabilities under the tri-level BYM models under a priori $\mathrm{P}(\mathrm{H} 0)$ : $25 \%$ (B); Posterior inclusion probabilities under the tri-level Poisson-Gamma under a priori $P(\mathrm{H} 0)$ : 75\% (C); Posterior inclusion probabilities under the tri-level BYM models under a priori P(H0): 75\% (D). Endometriosis, FVG, 2004-2017.

Table S2 (see Supplementary File S2) presents, as an appendix to Table 2, the distribution of endometriosis cases and population by age classes.

\subsection{Sensitivity Analysis}

Table S1 (Supplementary File S1-Part 1) shows a comparison between the case of endometriosis identified in the five high-risk municipalities and municipalities identified in the other areas of the region, based on the type of identification source and the limited number of demographic variables available from the data sources used. Overall, women in the five municipalities had a higher frequency of histological diagnosis of endometriosis (73.6\% vs. 53.8\%, $p<0.0001)$ and higher age at diagnosis. No difference was seen concerning the place of birth.

Comparisons with the regional average showed a negative association between anatomic pathology diagnosis and hospital discharge in the five high-risk municipalities. This suggests an ascertainment bias. Either the reporting was more careful than in the rest of the region, or many more pathology reports with a diagnosis of endometriosis were issued without accompanying hospital discharge records. Compared to the regional average, the relative risk observed in the five high-risk areas was 1.50 . Fixing a coverage probability equal to that of the five high-risk areas for the rest of the region, we had to increment the number of cases for the rest of the region, and the relative risk of the five high-risk areas would change from 1.50 to 1.31 (95\% CI 1.13; 1.52), (See Supplementary File S1-Part 2 for details in methods and results).

\section{Discussion}

Our study shows an estimated incidence of endometriosis consistent with those reported in similar registry-based studies, even if comparisons between studies are subject to limitations due to differences in settings and methodologies applied for case identification and definition. Concerning sampling surveys on endometriosis, in our study and in other similar registry-based ones, the operational definition of the incident case could lead to underestimating the number of disease cases $[4,11,12,14,45,46]$. 
The present study's strength relies on having performed a record-linkage among in/outpatients clinical and pathological databases and having conducted a follow-back to retrieve the date of incidence.

Geographical analysis showed a very strong spatial pattern with high-risk areas in the region's southeast part. When mapping disease risk, the aim is to estimate the risk distribution on a fine geographical resolution. The problem in meeting this goal is small areas are extremely heterogeneous in population denominators, resulting in difficulties in properly controlling for random variability. In spatial statistical literature, the term Disease Mapping refers to a collection of methods proposed to overcome such difficulties and "stabilize" or "smooth" the risk map. All these approaches rely on shrinkage estimators, and, among these, Bayesian estimators appear to be the most interesting [47].

Bayesian approaches to smoothing relative risk estimates may be misinterpreted as a solution to the problem of selective inference and multiple comparisons in Disease Mapping. However, estimation is a different task from testing. Multiple testing corrections based on $p$-values can be used to select high-risk areas. In a full Bayesian perspective classification, probabilities obtained from full Bayesian mixture models are adjusted for multiple comparisons and have the advantage of an easy way to perform sensitivity analysis on model assumptions. Both "frequentist" and full Bayesian analysis confirm a cluster of 5 adjacent municipalities in the FVG region, for a total of 296 cases of endometriosis (SIR $1.5 ; 90 \% \mathrm{CI}=1.36 ; 1.65)$ and 98 attributable cases $(90 \% \mathrm{CI}=71 ; 128)$.

An ascertainment bias cannot be excluded and could be geographically structured, contributing to explain the observed cluster of endometriosis incident cases. Indeed, a higher frequency of histological diagnosis was recorded in the five high-risk municipalities. This finding lends itself to two opposite interpretations. On the one hand, a more serious disease requiring increased diagnostic and surgical invasiveness. On the other hand, heightened attention to the ascertainment of endometriosis by gynecologists or pathologists working in the five areas is required. We used capture-recapture methods to evaluate for the presence of potential ascertainment bias. While our analyses suggest that there is some evidence of ascertainment bias, this is not enough to explain the observed cluster of cases in the five high-risk areas. We can thus conclude that the five areas are indeed at higher risk. Unfortunately, the very limited information available from the data sources used to identify women with endometriosis does not allow for a more detailed description of patients exposure and characteristics. The literature identifies several risk factors for endometriosis, including individual factors (i.e., shorter menstrual cycle length, low body mass index, lower parity, skin sensitivity and other somatic characteristics, Asian origin, autoimmune diseases, familiarity, genetics), behavioral factors (i.e., diet, physical activity, alcohol use, caffeine intake), and environmental pollution (i.e., dioxin, PCB, metals, phthalates) [48].

The most important Italian shipyard in the identified high-risk area is part of the largest shipbuilder in Europe. The company builds both commercial and military vessels. The area also comprises an oil and coal-powered energy plant ranking in the highest quartile of Italian energy plants and currently undergoing a heavily contested authorization renewal procedure (See Supplementary File S3-supplemental material, for further information on high-risk area).

In order to adequately address the role of the above-mentioned risk factors, an ad hoc study should be conducted that includes biomonitoring, evaluation of the individual and behavioral characteristics of the population, and life-course analysis of the exposures. Future studies will address these issues to explain the excess cases in these particularly environmentally stressed municipalities of FVG.

\section{Conclusions}

Our study is based on aggregated data at the municipality level. The main limitation of the study is that we cannot exclude a potential ecological bias. The statistical analysis by Bayesian spatially structured random effects should partially control for spatial confounding. The second limitation is that we cannot exclude a residual differential ascertainment 
bias among geographical units. We conducted a sensitivity analysis depicting a pessimistic scenario in which more attention is given to detecting endometriosis in the high-risk areas identified. Despite adopting these mitigation measures, we should be cautious in interpreting results from any geographical analysis, particularly when we lack information on spatially structured causal factors.

Generally, geographical variability in the occurrence of endometriosis is considered difficult to interpret because of lack of comparability in diagnosis, selection bias, and heterogeneity in study design across studies [49-51]. The use of geographic characteristics in the analysis was restricted to factors or characteristics not immediately interpretable as geographic-such as rural/urban areas, exposure to POPs, and so on $[52,53]$. When performed spatial analysis, results provide limited information due to potential ascertainment bias $[13,54]$. In our study, geographic analysis was the primary goal. We were especially careful to minimize differential ascertainment bias across geographical units and adopted sophisticated statistical methods to deal with multiple comparisons and selective inference. Spatial analysis and profiling of high-risk areas are useful tools to address environmental hypotheses in registry-based epidemiological studies.

Supplementary Materials: The following are available online at https:/ /www.mdpi.com/article/10 $.3390 /$ ijerph18137175/s1. Supplementary File S1, Title: Sensitivity analysis, Supplementary File S1 has two parts: Part 1: Comparing areas at higher/lower risk analysis (Table S1: Comparison between endometriosis cases identified in the five high-risk municipalities and those identified in the other areas of the region) and Part.2: Capture-recapture analysis; Supplementary File S2, Title: Appendix to Table 2 (Table S2: Distribution of endometriosis cases and population by age classes); Supplementary File S3, Title: Brief description of the high risk area.

Author Contributions: Conceptualization, A.B., F.B., G.R. and L.R.; Data curation, D.C. and M.G.; Formal analysis, D.C. and M.G.; Funding acquisition, L.R.; Investigation, D.C., M.G., A.B. and L.R.; Methodology, D.C. and M.G.; Project administration, A.B., F.B., G.R. and L.R.; Resources, D.C. and M.G.; Software, D.C. and M.G.; Supervision, A.B., F.B., G.R. and L.R.; Validation, A.B., F.B. and L.R.; Visualization, D.C., M.G., A.B., F.B., L.M., G.R., F.R., V.R., G.Z. and L.R.; Writing—original draft, D.C., M.G., A.B. and L.R.; Writing-review \& editing, D.C., M.G., A.B., F.B., L.M., G.R., F.R., V.R., G.Z. and L.R. All authors have read and agreed to the published version of the manuscript.

Funding: This project is supported by the Italian Ministry of Health with the 2018 Finalized Research Call [Project Code: RF-2018-12367534]. No involvement in study design, in the collection, analysis, and interpretation of data, in the writing of the report, and in the decision to submit the article for publication.

Institutional Review Board Statement: This study did not require ethical review and approval since data were extracted from the administrative databases of the Regional Repository of MicroData using an anonymous identifier.

Informed Consent Statement: Not applicable.

Data Availability Statement: The data underlying this article were provided by the FVG Regional Health Authority. Data will be shared on request to the corresponding author with the permission of the FVG Regional Health Authority.

Acknowledgments: We thank Francesca Buonomo, a gynecologist at the Institute for Maternal and Child Health-IRCCS "Burlo Garofolo", for helping us to correctly identify the interventions that allow the visualization of the endometrial tissue.

Conflicts of Interest: The authors declare no conflict of interest.

\section{References}

1. Bulun, S.E.; Yilmaz, B.D.; Sison, C.; Miyazaki, K.; Bernardi, L.; Liu, S.; Kohlmeier, A.; Yin, P.; Milad, M.; Wei, J. Endometriosis. Endocr. Rev. 2019, 40, 1048-1079. [CrossRef] [PubMed]

2. Zondervan, K.T.; Becker, C.M.; Koga, K.; Missmer, S.A.; Taylor, R.N.; Viganò, P. Endometriosis. Nat. Rev. Dis. Primers 2018, 4, 9. [CrossRef] 
3. As-Sanie, S.; Black, R.; Giudice, L.C.; Valbrun, T.G.; Gupta, J.; Jones, B.; Laufer, M.R.; Milspaw, A.T.; Missmer, S.A.; Norman, A.; et al. Assessing research gaps and unmet needs in endometriosis. Am. J. Obstet. Gynecol. 2019, 221, 86-94. [CrossRef]

4. Leibson, C.L.; Good, A.E.; Hass, S.L.; Ransom, J.; Yawn, B.P.; O’Fallon, W.M.; Melton, L.J. Incidence and characterization of diagnosed endometriosis in a geographically defined population. Fertil. Steril. 2004, 82, 314-321. [CrossRef]

5. Bulun, S.E. Endometriosis. N. Engl. J. Med. 2009, 360, 268-279. [CrossRef] [PubMed]

6. Nnoaham, K.E.; Hummelshoj, L.; Webster, P.; d'Hooghe, T.; de Cicco Nardone, F.; de Cicco Nardone, C.; Jenkinson, C.; Kennedy, S.H.; Zondervan, K.T.; Study, W.E. Impact of endometriosis on quality of life and work productivity: A multicenter study across ten countries. Fertil. Steril. 2011, 96, 366-373.e8. [CrossRef]

7. Simoens, S.; Dunselman, G.; Dirksen, C.; Hummelshoj, L.; Bokor, A.; Brandes, I.; Brodszky, V.; Canis, M.; Colombo, G.L.; DeLeire, T.; et al. The burden of endometriosis costs and quality of life of women with endometriosis and treated in referral centres. Hum. Reprod. 2012, 27, 1292-1299. [CrossRef] [PubMed]

8. Dunselman, G.A.; Vermeulen, N.; Becker, C.; Calhaz-Jorge, C.; D’Hooghe, T.; De Bie, B.; Heikinheimo, O.; Horne, A.W.; Kiesel, L.; Nap, A.; et al. ESHRE guideline: Management of women with endometriosis. Hum. Reprod. 2014, 29, 400-412. [CrossRef]

9. Rogers, P.A.; D’Hooghe, T.M.; Fazleabas, A.; Gargett, C.E.; Giudice, L.C.; Montgomery, G.W.; Rombauts, L.; Salamonsen, L.A.; Zondervan, K.T. Priorities for endometriosis research: Recommendations from an international consensus workshop. Reprod. Sci. 2009, 16, 335-346. [CrossRef]

10. Hsu, A.L.; Khachikyan, I.; Stratton, P. Invasive and noninvasive methods for the diagnosis of endometriosis. Clin. Obstet. Gynecol. 2010, 53, 413-419. [CrossRef]

11. Gylfason, J.T.; Kristjansson, K.A.; Sverrisdottir, G.; Jonsdottir, K.; Rafnsson, V.; Geirsson, R.T. Pelvic endometriosis diagnosed in an entire nation over 20 years. Am. J. Epidemiol. 2010, 172, 237-243. [CrossRef]

12. Ferrero, S.; Arena, E.; Morando, A.; Remorgida, V. Prevalence of newly diagnosed endometriosis in women attending the general practitioner. Int. J. Gynaecol. Obstet. 2010, 110, 203-207. [CrossRef] [PubMed]

13. Migliaretti, G.; Deltetto, F.; Delpiano, E.M.; Bonino, L.; Berchialla, P.; Dalmasso, P.; Cavallo, F.; Camanni, M. Spatial Analysis of the Distribution of Endometriosis in Northwestern Italy. Gynecol. Obstet. Invest. 2012, 73, 135-140. [CrossRef] [PubMed]

14. Houston, D.E.; Noller, K.L.; Melton, L.J., 3rd; Selwyn, B.J.; Hardy, R.J. Incidence of pelvic endometriosis in Rochester, Minnesota, 1970-1979. Am. J. Epidemiol. 1987, 125, 959-969. [CrossRef] [PubMed]

15. Buck Louis, G.M.; Hediger, M.L.; Peterson, C.M.; Croughan, M.; Sundaram, R.; Stanford, J.; Chen, Z.; Fujimoto, V.Y.; Varner, M.W.; Trumble, A.; et al. Incidence of endometriosis by study population and diagnostic method: The ENDO study. Fertil Steril. 2011, 96, 360-365. [CrossRef]

16. Hummelshoj, L.; Prentice, A.; Groothuis, P. Update on endometriosis. Womens Health (Lond) 2006, 2, 53-56. [CrossRef]

17. Missmer, S.A.; Hankinson, S.E.; Spiegelman, D.; Barbieri, R.L.; Marshall, L.M.; Hunter, D.J. Incidence of laparoscopically confirmed endometriosis by demographic, anthropometric, and lifestyle factors. Am. J. Epidemiol. 2004, 160, 784-796. [CrossRef]

18. Morassutto, C.; Monasta, L.; Ricci, G.; Barbone, F.; Ronfani, L. Incidence and Estimated Prevalence of Endometriosis and Adenomyosis in Northeast Italy: A Data Linkage Study. PLoS ONE 2016, 11, e0154227. [CrossRef]

19. Richardson, S.; Thomson, A.; Best, N.; Elliott, P. Interpreting posterior relative risk estimates in disease-mapping studies. Environ. Health Perspect. 2004, 112, 1016-1025. [CrossRef]

20. Storey, J.D. The positive false discovery rate: A Bayesian Interpretation and the Q-Value. Ann. Statist. 2003, 31, 2013-2035. [CrossRef]

21. Wakefield, J. Reporting and interpretation in genome wide association studies. Int. J. Epidemiol. 2008, 37, 641-653. [CrossRef]

22. Catelan, D.; Biggeri, A. Multiple testing in descriptive epidemiology. Geospat. Health 2010, 4, 219-229. [CrossRef]

23. Best, N.; Richardson, S.; Thomson, A. A comparison of Bayesian spatial models for disease mapping. Stat. Methods Med. Res. 2005, 14, 35-59. [CrossRef]

24. Militino, A.F.; Ugarte, M.D.; Dean, C.B. The use of mixture models for identifying high risks in disease mapping. Stat. Med. 2001, 20, 2035-2049. [CrossRef]

25. Biggeri, A.; Marchi, M.; Lagazio, C.; Martuzzi, M.; Böhning, D. Non parametric maximum likelihood estimators for disease mapping. Stat. Med. 2000, 19, 2539-2554. [CrossRef]

26. Muller, P.; Parmigiani, G.; Rice, K. FDR and Bayesian Multiple Comparisons Rules. In Proceedings of the Proc Valencia 2007/ISBA 8th World Meeting on Bayesian Statistics, Benidorm-Alicante, Spain, 1-6 June 2006; Oxford University Press: Oxford, UK.

27. Catelan, D.; Lagazio, C.; Biggeri, A. A hierarchical Bayesian approach to multiple testing in disease Mapping. Biom. J. 2010, 52, 784-797. [CrossRef] [PubMed]

28. ISTAT: The National Institute of Statistics. Available online: http:/ / www.demo.istat.it (accessed on 24 November 2020).

29. Breslow, N.E.; Day, N.E. Statistical Methods in Cancer Research-Volume II-The Design and Analysis of Cohort Studies; IARC Scientific Publications; Oxford University Press: Oxford, UK, 1987; Volume 82.

30. Clayton, D.; Kaldor, J. Empirical Bayes estimates of age-standardized relative risks for use in disease mapping. Biometrics 1987, 43, 671-681. [CrossRef]

31. Besag, J.; York, J.C.; Molliè, A. A Bayesian image restoration, with two applications in spatial statistics (with discussion). Ann. Inst. Statist. Math. 1991, 43, 1-20. [CrossRef]

32. Besag, J.; Kooperberg, G. On conditional and intrinsic autoregressions. Biometrika 1995, 82, 733-746. 
33. Best, N.G.; Arnold, R.A.; Thomas, A.; Waller, L.A.; Conlon, E.M. Bayesian models for spatially correlated disease and exposure data. In Bayesian Statistics 6; Bernardo, J.M., Berger, J.O., Dawid, A.P., Eds.; Oxford University Press: Oxford, UK, 1999; pp. 131-156.

34. Benjamini, Y.; Hochberg, Y. Controlling the false discovery rate: A practical and powerful approach to multiple testing. J. $R$ Statist. Soc. B 1995, 57, 289-300. [CrossRef]

35. Jones, H.E.; Ohlssen, D.I.; Spiegelhalter, D.J. Use of the false discovery rate when comparing multiple health care providers. J. Clin. Epidemiol. 2008, 61, 232-240. [CrossRef] [PubMed]

36. Goodman, S.N. Of P-Values and Bayes: A Modest Proposal. Epidemiology 2001, 12, 295-297. [CrossRef] [PubMed]

37. Edwards, W.; Lindman, H.; Savage, L.J. Bayesian statistical inference in psychological research. Psychol. Rev. 1963, 70, 193-242. [CrossRef]

38. Sellke, T.; Bayarri, M.J.; Berger, J.O. Calibration of $\mathrm{p}$ values for testing precise null hypotheses. Am. Stat. 2001, 55, 62-71. [CrossRef]

39. Scott, J.G.; Berger, J.O. An Exploration of Aspects of Bayesian Multiple Testing. J. Stat. Plan. Inference 2006, 136, $2144-2162$. [CrossRef]

40. Barbieri, M.M.; Berger, J.O. Optimal Predictive Model Selection. Ann. Stat. 2004, 32, 870-897. [CrossRef]

41. Lunn, D.J.; Thomas, A.; Best, N.; Spiegelhalter, D. WinBUGS-A Bayesian modelling framework: Concepts, structure, and extensibility. Stat. Comput. 2000, 10, 325-337. [CrossRef]

42. Gelman, A.; Rubin, D.B. Inference from iterative simulation using multiple sequences. Stat. Sci. 1992, 7, 457-511. [CrossRef]

43. Chao, A.; Tsay, P.K.; Lin, S.H.; Shau, W.Y.; Chao, D.Y. The applications of capture-recapture models to epidemiological data. Stat. Med. 2001, 20, 3123-3157. [CrossRef]

44. Egger, M.; Davey-Smith, G.; Altman, D. Systematic Reviews in Health Care: Meta-Analysis in Context, 2nd ed.; BMJ Pub. Group: London, UK, 2001.

45. Hyunkyung, K.; Minkyoung, L.; Hyejin, H.; Chung, Y.J.; Cho, H.H.; Yoon, H.; Kim, M.; Chae, K.H.; Jung, C.Y.; Kim, S.; et al. The Estimated Prevalence and Incidence of Endometriosis with the Korean National Health Insurance Service-National Sample Cohort (NHIS-NCS): A National Population-Based Study. J. Epidemiol. 2020. [CrossRef]

46. Rowlands, I.J.; Abbott, J.A.; Montgomery, G.W.; Hockey, R.; Rogers, P.; Mishra, G.D. Prevalence and Incidence of Endometriosis in Australian Women: A Data Linkage Cohort Study. BJOG 2020. [CrossRef]

47. Editorial: SMMR Special issue on disease mapping. Stat. Methods Med. Res. 2005, 14, 1-2. [CrossRef]

48. Shafrir, A.L.; Farland, L.V.; Shah, D.K.; Harris, H.R.; Kvaskoff, M.; Zondervan, K.; Missmer, S.A. Risk for and consequences of endometriosis: A critical epidemiologic review. Best Pract Res. Clin. Obstet. Gynaecol. 2018, 51, 1-15. [CrossRef] [PubMed]

49. Parazzini, F.; Roncella, E.; Cipriani, S.; Trojano, G.; Barbera, V.; Herranz, B.; Colli, E. The frequency of endometriosis in the general and selected populations: A systematic review. JEPPD 2020, 12, 176-189. [CrossRef]

50. Ghiasi, M.; Kulkarni, M.T.; Missmer, S.A. Is Endometriosis More Common and More Severe Than It Was 30 Years Ago? J. Minim. Invasive Gynecol. 2020, 27, 452-461. [CrossRef] [PubMed]

51. Bernuit, D.; Ebert, A.D.; Halis, G.; Strothmann, A.; Gerlinger, C.; Geppert, K.; Faustmann, T. Female perspectives on endometriosis: Findings from the uterine bleeding and pain women's research study. J. Endometr. 2011, 3, 73-85. [CrossRef]

52. Chapron, C.; Lang, J.H.; Leng, J.H.; Zhou, Y.; Zhang, X.; Xue, M.; Popov, A.; Romanov, V.; Maisonobe, P.; Cabri, P. Factors and Regional Differences Associated with Endometriosis: A Multi-Country, Case-Control Study. Adv. Ther. 2016, 33, $1385-1407$. [CrossRef]

53. Buck Louis, G.M.; Chen, Z.; Peterson, C.M.; Hediger, M.L.; Croughan, M.S.; Sundaram, R.; Stanford, J.B.; Varner, M.W.; Fujimoto, V.Y.; Giudice, L.C.; et al. Persistent lipophilic environmental chemicals and endometriosis: The ENDO Study. Environ. Health Perspect. 2012, 120, 811-816. [CrossRef]

54. Von Theobald, P.; Cottenet, J.; Iacobelli, S.; Quantin, C. Epidemiology of Endometriosis in France: A Large, Nation-Wide Study Based on Hospital Discharge Data. Biomed Res. Int. 2016, 2016, 3260952. [CrossRef] 